\section{Neuer Prognosemarker beim Leberzellkarzinom}

In einer Metaanalyse wurde untersucht, ob die Expression von Osteopontin als Prognosemarker für das Überleben von Patienten mit Leberzellkarzinom geeignet ist.

T rotz verbesserter Operationstechnik und perioperativem Management bleibt die Prognose von Patienten mit Leberzellkarzinom schlecht. Häufig treten Rezidive oder Lebermetastasen auf. Um geeignete therapeutische Strategien auswählen und das Überleben der Patienten verbessern zu können, wären Marker interessant, die eine Vorhersage von Rezidiven ermöglichen. Ein geeigneter Kan- didat wäre z.B. Osteopontin (OPN), das seit einigen Jahren mit der Entwicklung und Progression von Leberzellkarzinomen und anderen Tumoren in Verbindung gebracht wird. Bisherige Untersuchungen lieferten widersprüchliche Ergebnisse. Nun wurde der Zusammenhang zwischen der OPN-Überexpression und dem Überleben von Patienten mit hepatozellulärem Karzinom nun in einer Metaanalyse mit 1.158 Patienten untersucht. Eine hohe OPN-Expression geht mit einer signifikanten Reduktion von Gesamtüberleben ( $\mathrm{HR}=1,37)$ und krankheitsfreiem Überleben $(\mathrm{HR}=1,62)$ einher. Subgruppenanalysen zeigten zudem, dass die Assoziation zwischen hoher OPNExpression und verringertem Überleben auch für Patienten, die operiert wurden, sowie für Patienten ohne eine prä- oder postoperative adjuvante Therapie nachweisbar war. Eine Limitation der Studie: Die meisten Patienten stammten aus Asien. Die Autoren weisen darauf hin, dass Schlussfolgerungen aus diesen Daten mit Bedacht gezogen werden sollten.

Fazit: Bei Patienten mit hepatozellulärem Karzinom scheint eine hohe OPN-Expression mit einer schlechten Prognose einherzugehen.

Judith Neumaier

Zhang C-H et al. Prognostic significance of osteopontin in hepatocellular carcinoma: a metaanalysis. Int J Cancer. 2012;130(11):2685-92.

\title{
Metabolisches Syndrom steigert Risiko
}

\section{Die Inzidenz von Lebertumoren nimmt in den USA und in einigen europäischen Ländern zu. Etwa die Hälfte dieses Anstiegs lässt sich durch Hepatitis-C-Infektionen erklären. Gibt es weitere Faktoren?}

W elche Bedeutung metabolische Faktoren im Hinblick auf die Entwicklung primärer Leberkarzinome haben, wurde im prospektiven „Metabolic syndrome and cancer project" (Me-Can) mit 578.700 Personen aus Norwegen, Österreich und Schweden untersucht. In durchschnittlich zwölf Jahren Beobachtungsdauer wurden 266 primäre Leberkarzinome (hepatozelluläre oder intrahepatische Cholangiokarzinome) diagnostiziert.

Beim Vergleich der Quintilen zeigte sich, dass das Risiko für Leberkrebs mit zunehmendem BMI signifikant anstieg, mit steigendem Cholesterinspiegel signifikant abnahm. Patienten der obersten BMI-Quintile (im Mittel $31,3 \mathrm{~kg} / \mathrm{m}^{2}$ ) entwickelten fast doppelt so oft ein Leberkarzinom wie Patienten der untersten BMI-Quintile (im Mittel 20,7 kg/m²). Beim Blutdruck ergab sich zwar ein 2,8-faches Risiko für die oberste im Vergleich zur untersten Quintile, allerdings war das nach Berücksichtigung des BMI nicht mehr signifikant.

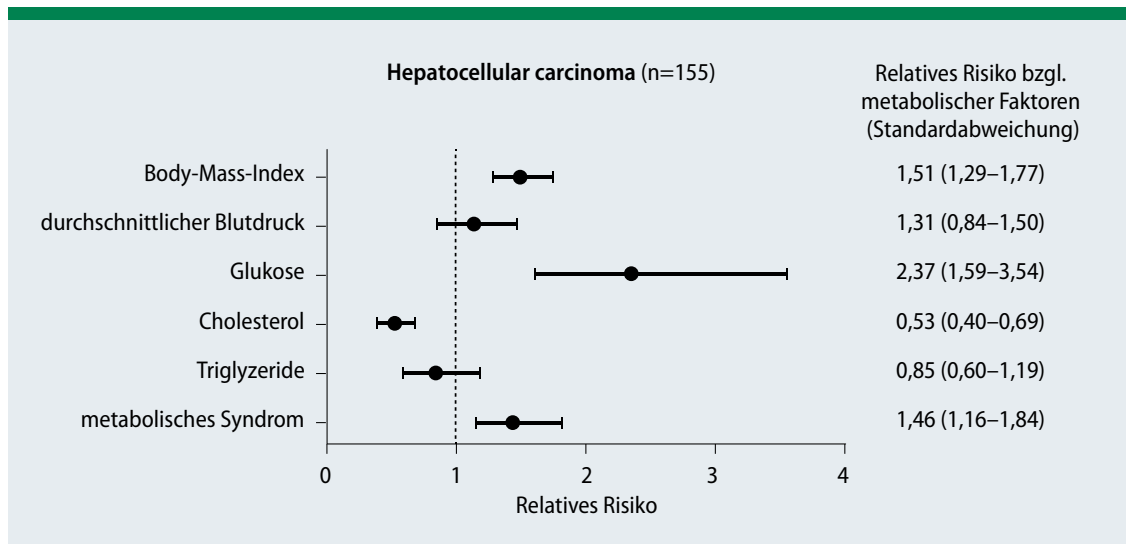

Blutzuckerspiegel, Body-Mass-Index und metabolisches Syndrom sind Risikofaktoren.
Zur Berechnung des relativen Risikos für ein Leberkarzinom wurde der standardisierte Summenscore des metabolischen Syndroms (Z-Score) herangezogen. Das relative Risiko für Leberkrebs pro Zunahme des Z-Scores um eine Einheit (korrigiert für Alter, Raucherstatus und BMI sowie stratifiziert nach Geburtsjahr, Geschlecht und Subkohorten) betrug $-1,39$ beim $\mathrm{BMI}$,

$-0,62$ beim Cholesterinspiegel sowie $-1,35$ beim metabolischen Syndrom.

Auch erhöhte Blutzuckerspiegel gingen mit einem größeren Leberkrebsrisiko einher. Aber nur für Männer ergab sich eine signifikante Korrelation (relatives Risiko: 2,67). Ursache für die negative Korrelation des Cholesterinspiegels mit dem Risiko für Leberkrebs könnten präklinische Tumoreffekte sein. So können Tumorzellen in der Leber Cholesterin akkumulieren.

Fazit: Wichtige metabolische Risikofaktoren wie der BMI und der Blutzuckerspiegel korrelieren mit dem Risiko für Leberkrebs. Erstmals wurde in dieser Studie auch nachgewiesen, dass das metabolische Syndrom in seiner Gesamtheit mit einer signifikanten Erhöhung des Leberkrebsrisikos einhergeht. Judith Neumaier

Borena W et al. Metabolic risk factors and primary liver cancer in a prospective study of 578,700 adults. Int J Cancer. 2012;131(1): 193-200. 